\title{
Classification of coronary artery tissues using optical coherence tomography imaging in Kawasaki disease
}

\section{Atefeh Abdolmanafi, Arpan Suravi Prasad, Luc Duong, Nagib Dahdah}

Atefeh Abdolmanafi, Arpan Suravi Prasad, Luc Duong, Nagib Dahdah, "Classification of coronary artery tissues using optical coherence tomography imaging in Kawasaki disease," Proc. SPIE 9786, Medical Imaging 2016: Image-Guided Procedures, Robotic Interventions, and Modeling, 97862U (18 March 2016); doi: 10.1117/12.2216943 


\title{
Classification of coronary artery tissues using Optical Coherence Tomography imaging in Kawasaki disease
}

\author{
Atefeh Abdolmanafi ${ }^{1 \mathrm{a}}$, Arpan Suravi Prasad ${ }^{\mathrm{a}}$, Luc Duong ${ }^{\mathrm{a}}$, Nagib Dahdah ${ }^{\mathrm{b}}$ \\ ${ }^{a}$ Dept. of Software and IT Engineering, École de technologie supérieure, Montréal, Canada \\ ${ }^{\mathrm{b}}$ Div. of Pediatric Cardiology, and Research Center, Centre Hospitalier Universitaire Sainte-Justine, \\ Montréal, Canada
}

\begin{abstract}
Intravascular imaging modalities, such as Optical Coherence Tomography (OCT) allow nowadays improving diagnosis, treatment, follow-up, and even prevention of coronary artery disease in the adult. OCT has been recently used in children following Kawasaki disease (KD), the most prevalent acquired coronary artery disease during childhood with devastating complications. The assessment of coronary artery layers with OCT and early detection of coronary sequelae secondary to KD is a promising tool for preventing myocardial infarction in this population. More importantly, OCT is promising for tissue quantification of the inner vessel wall, including neo intima luminal myofibroblast proliferation, calcification, and fibrous scar deposits. The goal of this study is to classify the coronary artery layers of OCT imaging obtained from a series of KD patients. Our approach is focused on developing a robust Random Forest classifier built on the idea of randomly selecting a subset of features at each node and based on second- and higher-order statistical texture analysis which estimates the gray-level spatial distribution of images by specifying the local features of each pixel and extracting the statistics from their distribution. The average classification accuracy for intima and media are $76.36 \%$ and $73.72 \%$ respectively. Random forest classifier with texture analysis promises for classification of coronary artery tissue.
\end{abstract}

Keywords: Optical coherence tomography, Tissue classification, Coronary artery, Kawasaki disease, Image-guided interventions

\section{INTRODUCTION}

Kawasaki disease (KD) is an acute childhood disease characterized by fever, rash, bilateral nonexudative conjunctivitis, erythema of the lips and oral mucosa, changes in the extremities, and cervical lymphadenopathy. In about $15 \%$ to $25 \%$ of untreated children, there is a risk of coronary artery aneurysms or ectasia, which may be followed by intimal thickening, thrombi, stenosis, lamellar calcifications, disappearance of the media border, and significantly the stiffness of the arterial wall. They can lead to myocardial infarction and even death if they are not diagnosed and properly treated[1]. Coronary arteries are responsible to convey oxygen-rich blood to the myocardium. Therefore, cardiac function depends strongly on the coronary blood flow to the myocardium and subsequently, the functionality of cardiac tissues. In vivo intravascular visualization of coronary arteries is a challenging task, especially in paediatric patients, where the diameters of vessels are smaller and the heart rate is higher compared to the adult[2]. Nevertheless, OCT has been recently used to image coronary artery layers in a series of KD pediatric patients, as young as 3.2 years old, with various levels of injury and sequelae[3].

Optical Coherence Tomography (OCT) is promising to quantify the inner parts of the vessels by using near-infrared light to produce a sequence of intracoronary images with high resolution ranging from 10-20 $\mu \mathrm{m}$. Inner vessel wall geometry allows detecting and evaluating biophysical and dynamic properties of arterial wall, thickness of coronary artery layers, and various thrombi and calcifications. In order to obtain a sequence of cross-sectional images of coronary artery, the catheter is advanced from patient's femoral artery to the distal end of coronary artery via a guiding catheter. Images are produced by automated pull-back the probe with constant speed of around 0.5 to $3.0 \mathrm{~mm} / \mathrm{s}$ through the catheter[4, 5].

1. Atefeh.abdolmanafi.1@ens.etsmtl.ca; phone: (514) 396-8800 ext. 7817

Medical Imaging 2016: Image-Guided Procedures, Robotic Interventions, and Modeling,

edited by Robert J. Webster III, Ziv R. Yaniv, Proc. of SPIE Vol. 9786, 97862U

(C) 2016 SPIE · CCC code: 1605-7422/16/\$18 · doi: 10.1117/12.2216943 
Since OCT is a new intravascular modality there are no specific segmentation method available. Most of the segmentation and classification techniques have been applied on Intravascular Ultrasound (IVUS) images. Unal et al.[6] described a segmentation method performed by the minimization of an energy function using non-parametric probability densities, and employing edge information to segment media-adventitia. Shape-driven method is not successful when there are strong calcifications or large side branch openings and also edge information is not reliable in sequence of images. Another successful approach on IVUS images has been proposed by Mendizabal-Ruize et al.[7]. The base of this technique is to minimize a probabilistic cost function, which describes a likelihood field according to the probability of each pixel belonging to blood or non-blood regions using SVM as the classifier. This approach is more stable compared with other segmentation methods but it is just able to detect the luminal border. Several groups have been focused on intravascular OCT images to characterize three major coronary atherosclerotic plaques, which have been demonstrated as fibrous, calcified, and lipid-rich plaques[8,9]. They mainly have measured the optical properties of tissue to do the classification. But, none of those above works are focused on tissue quantification in pediatric patients. Also, in the case of Kawasaki disease, thickness of intima and disappearance of media border are two significant issues that require information about intima and media layers.

In this study, our approach is focused on developing a robust machine learning approach to train a classifier to discriminate between coronary artery tissues based on second- and higher-order statistical texture analysis which estimates the gray-level spatial distribution of images by specifying the local features of each pixel and extracting the statistics from their distribution. This work is organized as follows: Section 2 presents the pre-processing step to detect the lumen boundaries from OCT images, texture analysis, and the training of a classifier to discriminate between coronary tissues. Section 3 reports and discusses the results obtained on OCT patient datasets. Section 4 concludes by contribution of the current work and future work plans.

\section{METHODS}

\subsection{Data collection}

Intracoronary cross-sectional images are obtained during selective coronary catheterization using the ILUMIEN OCT system (St. Jude Medical Inc., St. Paul, Minnesota, USA). Each image is a sequence of DICOM images 270 frames for each patient. The lumen is the inner part of the artery which blood flows through it, which is surrounded by an arterial wall composed of three layers. In the normal coronary structure, the inner-most and the first signal-rich homogenous layer in OCT image, the intima, is comprised by endothelial cells in direct contact with circulating blood. Briefly, the intima is a transparent and achromatic structure and is extremely elastic. The media is the central layer of the vessel and is composed of smooth muscle cells, lined by the inner and the outer elastica layers composed of elastic fibers. The media is characterized as a thin signal-poor band in OCT images. Finally, the adventitia is the outermost layer of the artery which encompasses the media and is determined as signal-rich layer in OCT images[10].

\subsection{Pre-processing}

First, our analytical processing starts with an automatic recognition of the guide-wire, removed from the images, and a Gaussian filter is applied to smoothen the catheter. Then, an active contour segmentation removes unwanted red blood cells by calculating the fraction of non-connected elements. High heart rate in children, heart pulsation, and patient respiration during the imaging process are the factors, which affect the catheter motion. Therefore, the catheter does not move through the centroid of the lumen and the center of the cross-sectional images varies from frame to frame. The approximate center of the cross-sections is calculated as the centroid of the contours and the images were mapped to planar by transferring all the points from Cartesian coordinates to planar representation in polar coordinates. Planar representation offers two main advantages: first, each column represents a sample over the vessel's radius and second, since the vessel is a circular closed shape, the contour has to be continuously connected over the full range between 0 359 degrees. Also, the inner border of intima was detected by moving the approximate center of the contour in all directions of the image and tracing the first white point from center in all angular displacements (Figure 1).

The pre-processing was performed step by step on all the frames of a sequence for each patient and the results were saved. After converting the images to planar, the intima and media layers were recognized, manually classified, and saved as a sequence for each patient. 


\subsection{Texture analysis}

Feature extraction in this study is based on texture analysis that was used to study the spatial alignment of intensity variation in an image. Texture-based statistical approaches are known as the simplest and more accurate techniques, which estimate the gray-level spatial distribution of an image by specifying the local features of each pixel and extracting the statistics from their distribution. Gray-Level Co-occurrence Matrix (GLCM) and Gray-Level Run length Matrix (GLRM) are examples of second- and higher-order methods respectively, which consider the spatial relationships among pixels in different orientations. The co-occurrence matrix is extracted from a gray-level image and demonstrates the frequency of occurring a pixel with value $i$ in the vicinity of pixels with values $j$ through the horizontal, vertical, or diagonal directions specified by a given offset. Contrast, homogeneity, correlation, and energy are the most significant parameters, which are useful in classification tasks and can be extracted from GLCM. Contrast is defined as the local gray-level changes in GLCM. Homogeneity measures the uniformity of the non-zero values of GLCM; the lower homogeneity means the higher gray-level variations and consequently the higher contrast. Correlation calculates the joint probability of occurring particular pixel pairs in gray-level and energy is the measure of uniformity of the texture, it shows the local homogeneity. Therefore, when energy is high, the homogeneity will also be high. The Gray-Level Run length Matrix is calculated based on the higher-order statistical texture information. A set of linearly connected pixels with the same gray-level is defined as gray-level run. The probability of occurrence of runs with length $\mathrm{j}$ at gray-level $\mathrm{i}$ and angle $\theta$ is given by $P(i, j \mid \theta$ ). Short-Run Emphasis (SRE), Long-Run Emphasis (LRE), Gray-Level Non-Uniformity for Run (GLNUR), Run-Length Non-Uniformity (RLNU), Run Percentage (RP), Low Gray-Level Run Emphasis (LGRE), and High Gray-Level Run Emphasis (HGRE) were selected as the higher-order statistical features extracted from GLRM[11, 12].
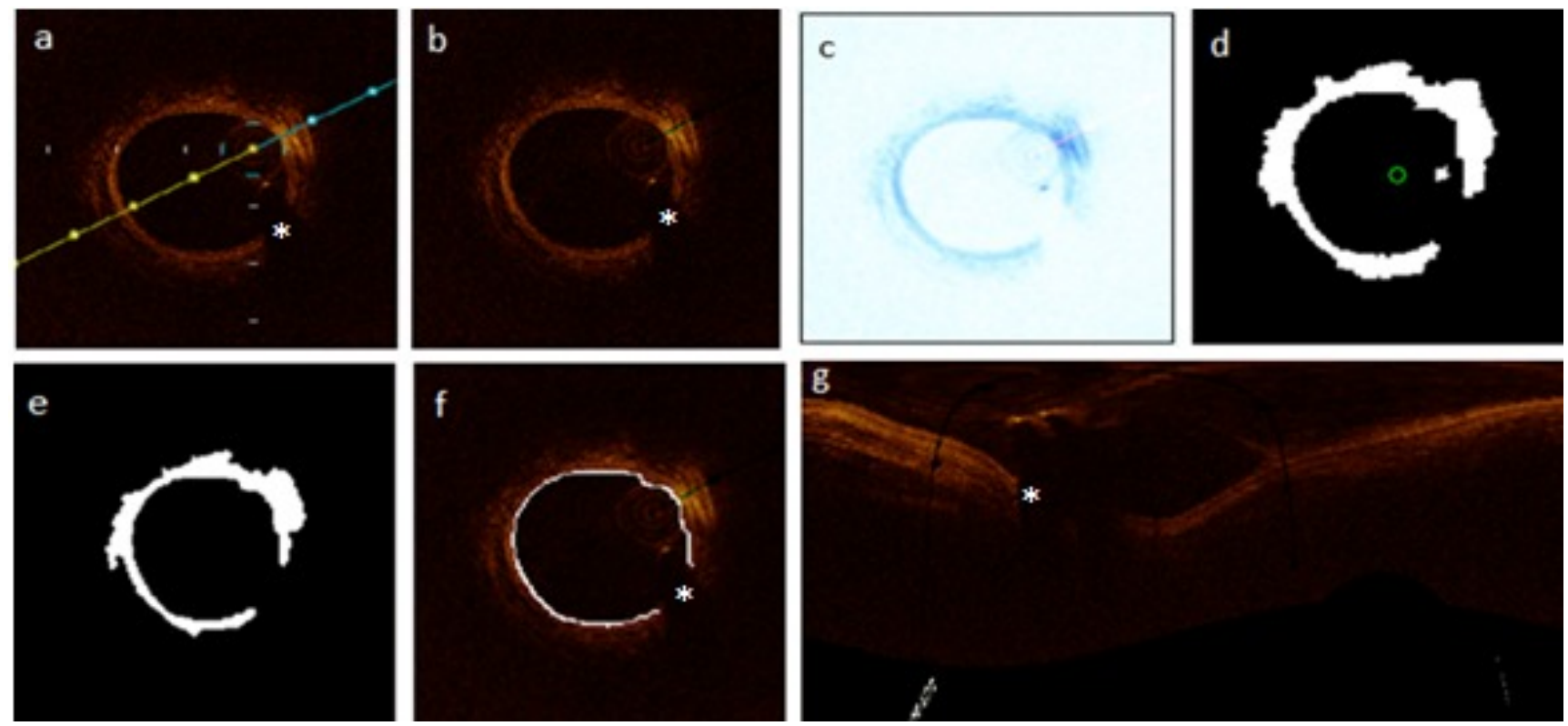

Figure 1.Pre-processing procedure from the OCT images. (a) Original cross-sectional image obtained from OCT system during the catheterization. (b) After detection and removal of the guide-wire. (c) Gaussian filtering to smoothen the catheter. (d) Cross-sectional image after applying active contour. Removing of unwanted red blood cells intima border detection, and rectangular representation of the image are illustrated in images (e), (f), and (g) respectively. Asterisk shows guide-wire shadowing artifact.

\subsection{Developing Random Forest for tissue classification}

Random Forest expands on the idea of randomly selecting a subset of features at each node in the learning process. This is done to reduce the correlation among the final trees. At each node, a small group of features were chosen to split on; the trees were grown based on CART methodology to maximum size without pruning. The number of randomly selected predictors $\left(\mathrm{m}_{\mathrm{try}}\right)$ and the number of trees are two tuning parameters of Random Forest classifier. There is a low risk of overfitting in Random Forest; hence, we can increase the number of trees gradually if the performance of the forest improves. But, the less number of trees, the less computational burden to create the forest. Hence, we set $\mathrm{m}_{\text {try }}$ to 5 and increased the number of trees up to 100 to be computationally faster for this part of the work. Random Forest is 
interesting compared to other algorithms, since it runs efficiently on large data sets and it is able to handle a large number of input variables. To deal with noisy data, which is the main challenge of working with medical images, Random Forest is a robust classifier because each learner is chosen independently from the previous ones. Estimating the effective variables in classification and missing data are other advantages of this method[13].

\subsection{Training and validation}

To train the classifier, we started with 15 frames from the beginning of the OCT sequence. For each frame, the pixels were labeled according to the manual segmentation as 1 for intima, 2 for media, and 0 for other parts of the image including the guide-wire shadow, lumen, catheter, adventitia and the whole background. After labeling, the next step is to extract the features corresponding to each labeled region. The GLCMs and GLRMs created based on the spatial relationship of each pixel of interest and its adjacent pixel in different orientations of $0,45,90$, and 135 degrees. Then, for each angle of image coordinate system, the statistics derived along the radius from GLCMs and GLRMs separately and combined together as a single vector of features for each label. Then, the feature matrix for each frame was created by repeating the second step for all the angles from 0-359 degrees. The first and second steps were repeated recursively for all the 15 frames and the final feature matrix built on the statistics derived from all the 15 frames used for training.

Classification accuracy is calculated within intima and media separately by comparing the ground-truth samples, obtained from the manual segmentation, to the automated classification results. Per-class accuracy for intima and media is reported as the percentage of the samples correctly predicted as belonging to desire class divided by the whole number of samples predicted in that class. Overall accuracy is computed as the percentage of the samples assigned to the correct intima and media labels divided by the whole samples were predicted as belonging to both intima and media.

\section{RESULTS AND DISCUSSION}

Examples of the original images for continuous frames of each sequence were illustrated in Figures 2(a), 3(a), and 4(a). Figures 2(b), 3(b), and 4(b) show the corresponding classification results for both intima (red label map) and media (green label map) obtained from the proposed algorithm. The images were manually segmented to create a set of groundtruth, which are shown in Figures 2(c), 3(c), and 4(c). Per-class and overall accuracy is reported in Table 1 for the three frames of each sequence, which are shown in figures 2,3 , and 4 . The average per-class classification accuracies for all three sequences are computed which are $76.36 \%$ and $73.72 \%$ for intima and media respectively.

The algorithm is validated using manual segmentation and good per-class accuracy obtained from the proposed methods. Because of the small size of coronary arteries and high heart rate of young children and infants during the imaging process, diagnostic assessment of coronary artery abnormalities is a very challenging task in pediatric cardiology. On the other hand, image acquisition in realistic clinical imaging conditions leads to different OCT artifacts including residual blood which can reduce the tissue brightness, non-uniform rotational distortion as a result of changes in optical fiber rotational speed, sew-up artifact caused by fast movements of imaging wire or artery, artifacts related to eccentric wire position, side branches and large vessels. Considering imprecise evaluation and imperfect measurements as a result of all mentioned issues that might occur during the imaging process, our results are promising and indicate the ability of our technique to detect intima and media layers with high accuracy and less computational complexity. Nevertheless, the advantage of OCT over IVUS, in terms of tissue quantification, is the fact that infrared light penetrates across calcium deposits in OCT rendering deeper tissue available for analysis. Finally, to our knowledge, this is the first study to quantify coronary artery tissues in pediatric patients, including OCT derived imaging. 

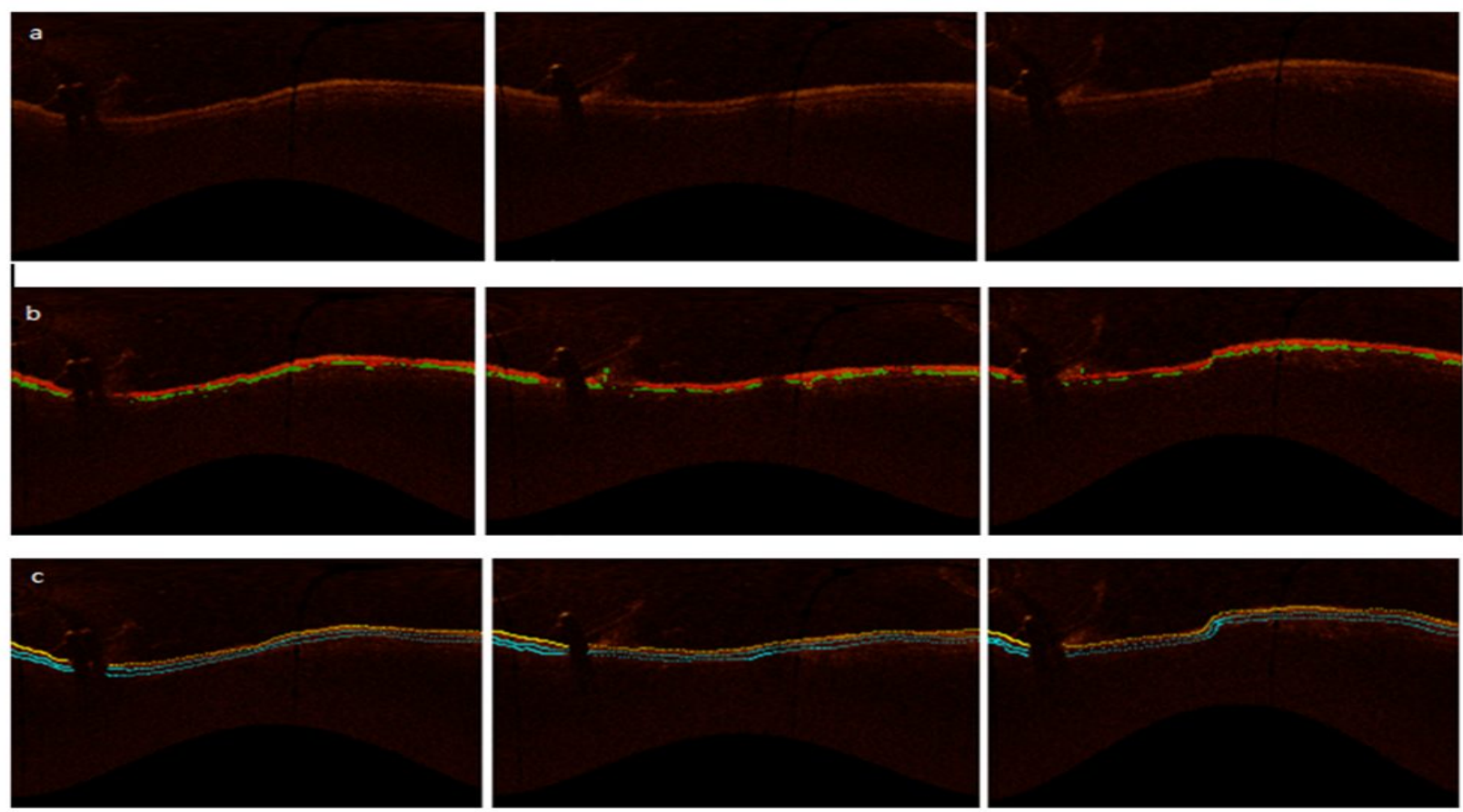

Figure 2.Tissue classification results on OCT images of coronary artery for three consecutive frames of a sequence of intracoronary images for first patient. (a) Original images in rectangular domain. (b) Tissue classification results for intima and media layers of coronary artery. (c) Manual segmentation.
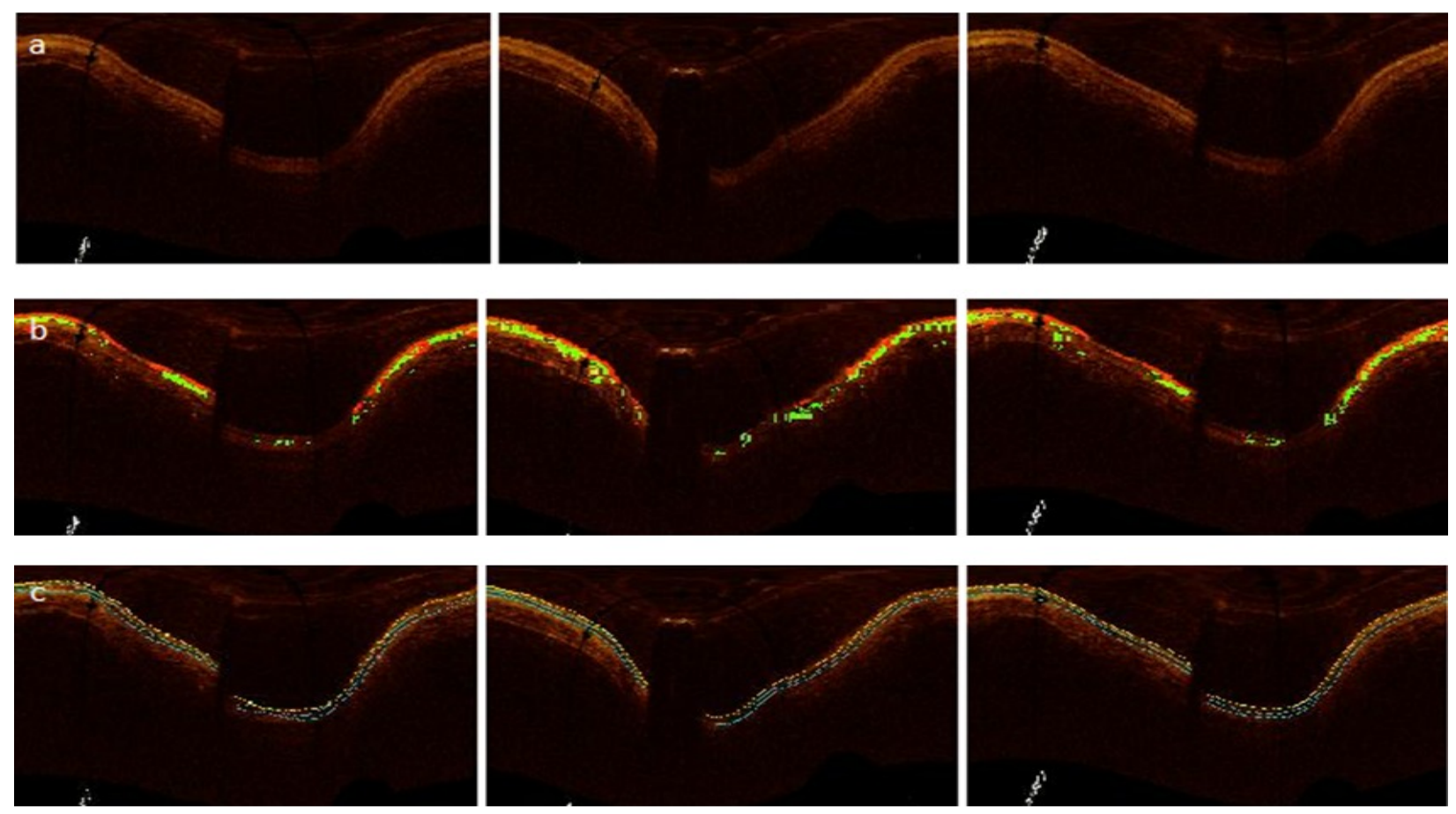

Figure 3.Tissue classification results on OCT images of coronary artery for three consecutive frames of a sequence of intracoronary images for second patient. (a) Original images in rectangular domain. (b) Tissue classification results for intima and media layers of coronary artery. (c) Manual segmentation. 

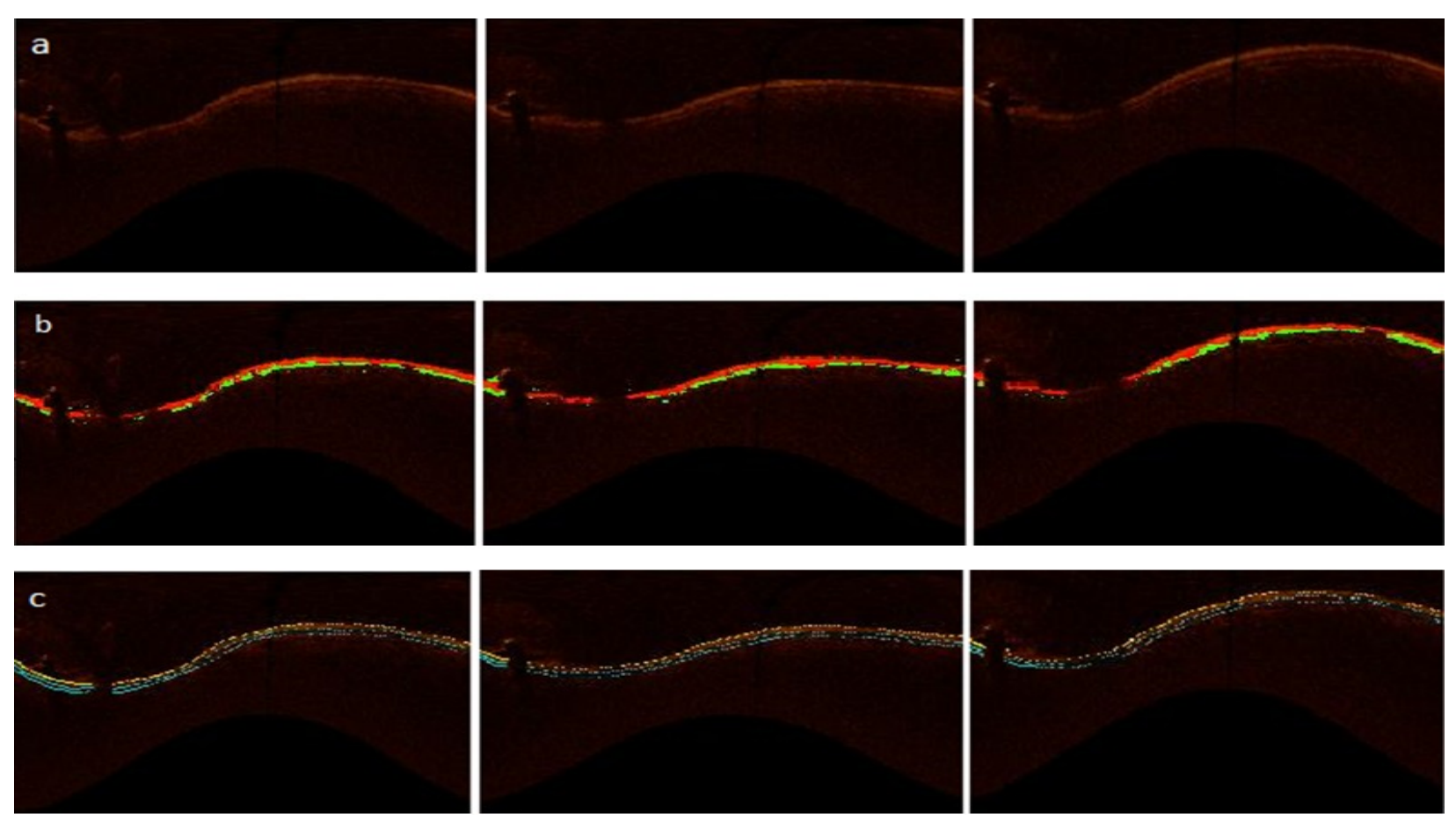

Figure 4.Tissue classification results on OCT images of coronary artery for three consecutive frames of a sequence of intracoronary images for third patient. (a) Original images in rectangular domain. (b) Tissue classification results for intima and media layers of coronary artery. (c) Manual segmentation.

\begin{tabular}{|l|l|c|c|c|}
\hline \multicolumn{2}{|c|}{} & Patient 1 & Patient 2 & Patient 3 \\
\hline Per-class accuracy & Intima & $75.3948 \pm 2.1860$ & $70.9996 \pm 1.0168$ & $80.0339 \pm 4.1348$ \\
\cline { 2 - 5 } & Media & $73.5365 \pm 2.6965$ & $70.5675 \pm 5.0535$ & $73.0390 \pm 8.0118$ \\
\hline Overall accuracy & Intima and media & $74.4058 \pm 2.1062$ & $70.169 \pm 4.0069$ & $77.5956 \pm 1.4932$ \\
\hline
\end{tabular}

Table 1.Accuracy of coronary artery tissue classification using texture-based Random Forest. Per-class accuracy is reported as the percentage of the samples correctly predicted as belonging to desire class divided by the whole number of samples predicted in that class. Overall accuracy is computed as the percentage of the samples assigned to the correct intima and media labels divided by the whole samples were predicted as belonging to both intima and media.

\section{CONCLUSION}

We developed an automated tissue classification algorithm to classify coronary artery tissues in pediatric patients with the history of Kawasaki disease. The results confirm the robustness of the method considering OCT artifacts and intravascular imaging challenges in young children and infants. This will contribute to a robust method in pediatric cardiology to estimate intima-media thickening, evaluate the functionality of coronary arteries, and reduce the risk of sudden death in patients with Kawasaki disease.

Further experiments should be focused on improving the precision of the method during the learning process, training, and testing the classifier on intracoronary OCT images obtained from more patients. In the case of KD, there is a neointima proliferation but the stiffness of the tissue is abnormal for various reasons, including media and elastic destruction, calcium deposits and fibrous scarring, which affects vascular elasticity and distensibility. Detecting other abnormalities and evaluating the dynamic and geometry of vessel is another supplementary approach to quantify the severity of KD using stationary OCT image acquisition.

\section{ACKNOWLEDGEMENT}

This study was funded by NSERC Discovery Grant program and Fonds de recherché du Quebec Nature et Technologyies. 


\section{REFERENCES}

[1] Orenstein, J.M., et al., Three linked vasculopathic processes characterize Kawasaki disease: a light and transmission electron microscopic study. PloS one, 2012.

[2) Newburger, J.W., et al., Diagnosis, treatment, and long-term management of Kawasaki disease a statement for health professionals from the Committee on Rheumatic Fever, Endocarditis and Kawasaki Disease, Council on Cardiovascular Disease in the Young, American Heart Association. Circulation, 2004. 110(17): p. 2747-2771.

[3] Dionne, A., et al., Coronary Wall Structural Changes in Patients With Kawasaki Disease: New Insights From Optical Coherence Tomography (OCT). Journal of the American Heart Association, 2015.

[4] Bezerra, H.G., et al., Intracoronary optical coherence tomography: a comprehensive review: clinical and research applications. JACC: Cardiovascular Interventions, 2009. 2(11): p. 1035-1046.

[5] Ferrante, G., et al., Current applications of optical coherence tomography for coronary intervention. International journal of cardiology, 2013. 165(1): p. 7-16.

[6] Unal, G., et al., Shape-driven segmentation of the arterial wall in intravascular ultrasound images. Information Technology in Biomedicine, IEEE Transactions on, 2008. 12(3): p. 335-347.

[7] Mendizabal-Ruiz, E.G., M. Rivera, and I.A. Kakadiaris, Segmentation of the luminal border in intravascular ultrasound B-mode images using a probabilistic approach. Medical image analysis, 2013. 17(6): p. 649-670.

[8] Gargesha, M., et al., Parameter estimation of atherosclerotic tissue optical properties from threedimensional intravascular optical coherence tomography. Journal of Medical Imaging, 2015. 2(1): p. 016001-016001.

[9] Van Soest, G., et al., Atherosclerotic tissue characterization in vivo by optical coherence tomography attenuation imaging. Journal of biomedical optics, 2010. 15(1): p. 011105-011105-9.

[10] Ligthart, J., N. Bruining, and G. van Soest, The diagnostic value of intracoronary optical coherence tomography. Herz, 2011.36(5): p. 417-429.

[11] Aggarwal, N. and R. Agrawal, First and second order statistics features for classification of magnetic resonance brain images. 2012.

[12] Albregtsen, F., B. Nielsen, and H.E. Danielsen. Adaptive gray level run length features from class distance matrices. in Pattern Recognition, 2000. Proceedings. 15th International Conference on. 2000. IEEE.

[13] Criminisi, A. and J. Shotton, Decision forests for computer vision and medical image analysis. 2013: Springer Science \& Business Media. 